\title{
Kriterien der Wissenschaftlichkeit
}

Peter Schulthess

Psychotherapie soll wissenschaftlich fundiert und evidenzbasiert sein. Das ist eine Forderung, die in den Schweizer Akkreditierungsverfahren überprüft und von ExpertInnen der Agentur für Akkreditierung und Qualitätssicherung (AAQ) und der Psychologieberufekommission (PsyKo) unterschiedlich bzw. uneinheitlich gehandhabt wurde, da verbindliche Kriterien zur Feststellung, was genügend evidenzbasiert sei und was nicht, weder aus dem Psychologieberufegesetz (PsyG) noch aus der Akkreditierungsverordnung ableitbar sind. Im PsyG heisst es lediglich: Die Weiterbildung «basiert auf den aktuellen wissenschaftlichen Erkenntnissen im Fachgebiet» (PsyG, Art. 5, Abs. 1). Im Anhang der Akkreditierungsverordnung steht: «Die Weiterbildung vermittelt umfassendes, wissenschaftlich fundiertes und empirisch gesichertes Wissen und Können» (AkkredV-PsyG, Art. 3.1.a), «Die Inhalte der Weiterbildung entsprechen dem aktuellen Wissensstand im Fachgebiet» (ebd., Art. 3.1.b), sowie «Die Weiterbildung vermittelt mindestens ein umfassendes, theoretisch und empirisch fundiertes Modell des psychischen Erlebens, des Verhaltens, der Entstehung und des Verlaufs psychischer Störungen und Krankheiten sowie des psychotherapeutischen Veränderungsprozesses» (ebd., Art. 3.3.a).
Welche Kriterien erfüllt sein müssen, um einen Therapieansatz als empirisch fundiert gelten $\mathrm{zu}$ lassen, wurde wohl bewusst offen gelassen, da diese in der Fachwelt bekannterweise sehr umstritten sind. Das führte im Laufe der Akkreditierungsverfahren aber je nach ExpertInnenzusammensetzung dazu, dass Bezüge zu den Richtlinien des deutschen Wissenschaftlichen Beirates Psychotherapie (WBP) oder der American Psychological Association (APA) hergestellt wurden. Immer mit der bedauernden Feststellung, dass jene mangels gesetzlicher Grundlage in diesen Akkreditierungsverfahren nicht angewandt werden könnten und deshalb ein erweiterter bzw. «weicherer» Wissenschaftlichkeitsbegriff anzuwenden sei.

Welcher Art Studien sein müssen, um als Beleg für die Wirksamkeit eines Verfahrens gelten zu können, ist sehr umstritten und wird heftig in der Welt der Psychotherapieforschung diskutiert - in der APA, in der Society for Psychotherapyresearch (SPR) und in Deutschland, wo jüngst zwei Gutachten des WBP zur Humanistischen Therapie und zur Gestalttherapie hohe Wellen schlugen und dem WBP harsche Kritik einbrachten (vgl. Schulthess, 2018a, b).

Der Streit dreht sich auch hier um die Frage, wer bestimmen kann, welche Art von Studien einen
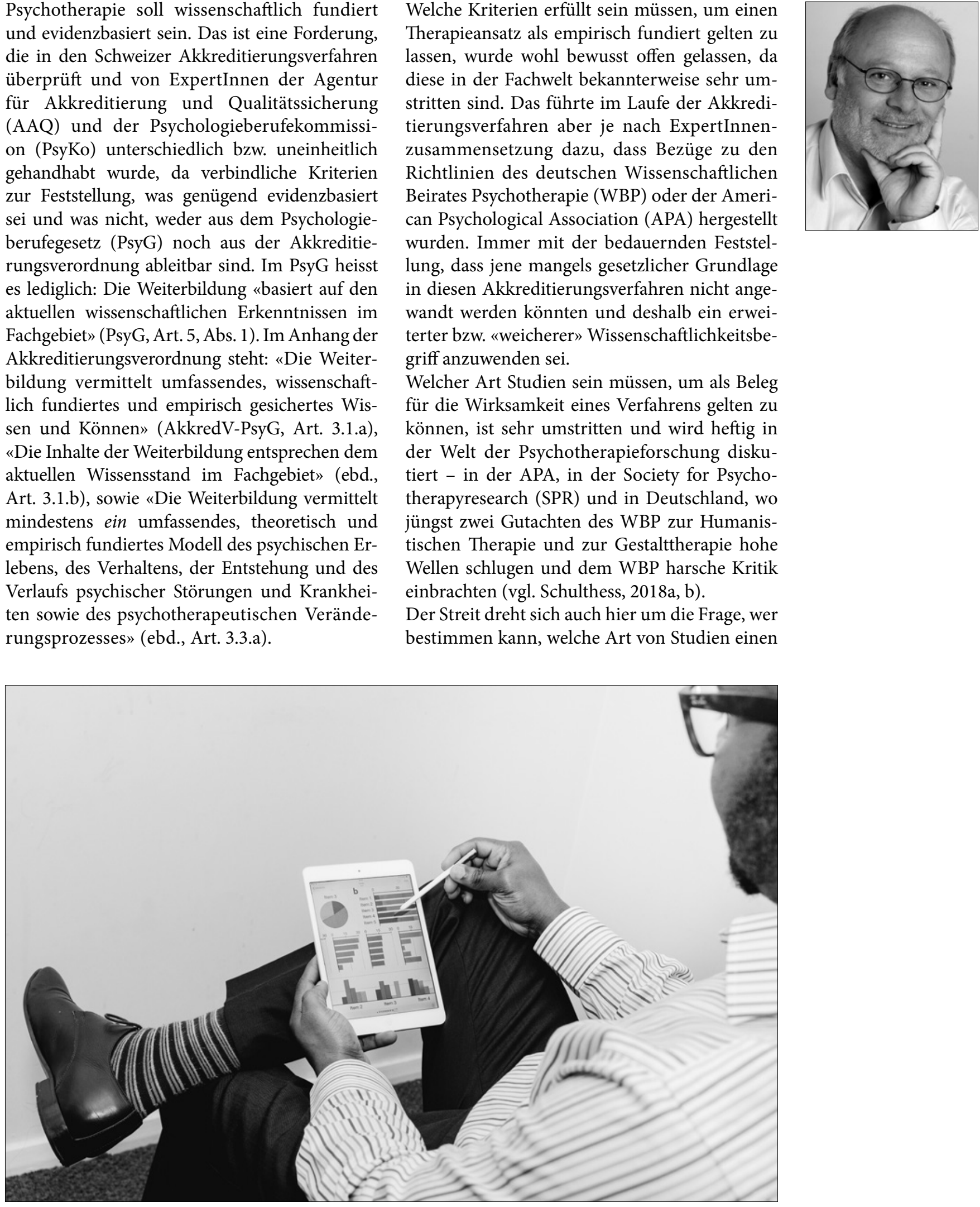
hinreichenden Wirksamkeitsnachweis begründen können. Der WBP hat sich in einem Methodenpapier (WBP, 2010) selbst einen Kriterienkatalog gegeben, den er im Lauf der Zeit verschärfte und faktisch nur noch randomisierte kontrollierte Studien (randomized controlled trial; RCT) anerkannte, obwohl er im Methodenpapier schrieb, dass auch andere Studien zu berücksichtigen seien. Alle übrigen Studien wurden als methodisch ungenügend abgewiesen. Das brachte ihm die Kritik ein, sich weder am internationalen wissenschaftlichen Diskurs $\mathrm{zu}$ orientieren, noch die eigenen Kriterien wirklich anzuwenden. Gemäss diesem Methodenpapier kann ein Verfahren nur dann als solches bzw. als einer Grundorientierung zugehörig anerkannt werden, wenn es eine kohärente Theorie aufweist. Zum Nachweis der Wirksamkeit müssen pro Störungsbereich mindestens drei RCTs vorliegen, eine davon mit Katamnese. Aufgrund seines Status ist der WBP befugt, darüber zu befinden, was anerkennenswert ist und was nicht. Das ist ein bedeutsamer Unterschied zum Status der PsyKo in der Schweiz, die nicht entscheiden, sondern laut PsyG lediglich beratend wirken kann. Der Entscheid liegt in der Schweiz beim Eidgenössischen Departement des Innern (EDI). Es ist nicht an die Empfehlung der PsyKo gebunden und kann deshalb auch gegen deren Rat entscheiden, was bei den Akkreditierungsverfahren öfter geschah: Das EDI richtete sich in der Regel nach den Empfehlungen der AAQ und nicht denen der PsyKo, wenn diese nicht übereinstimmten.

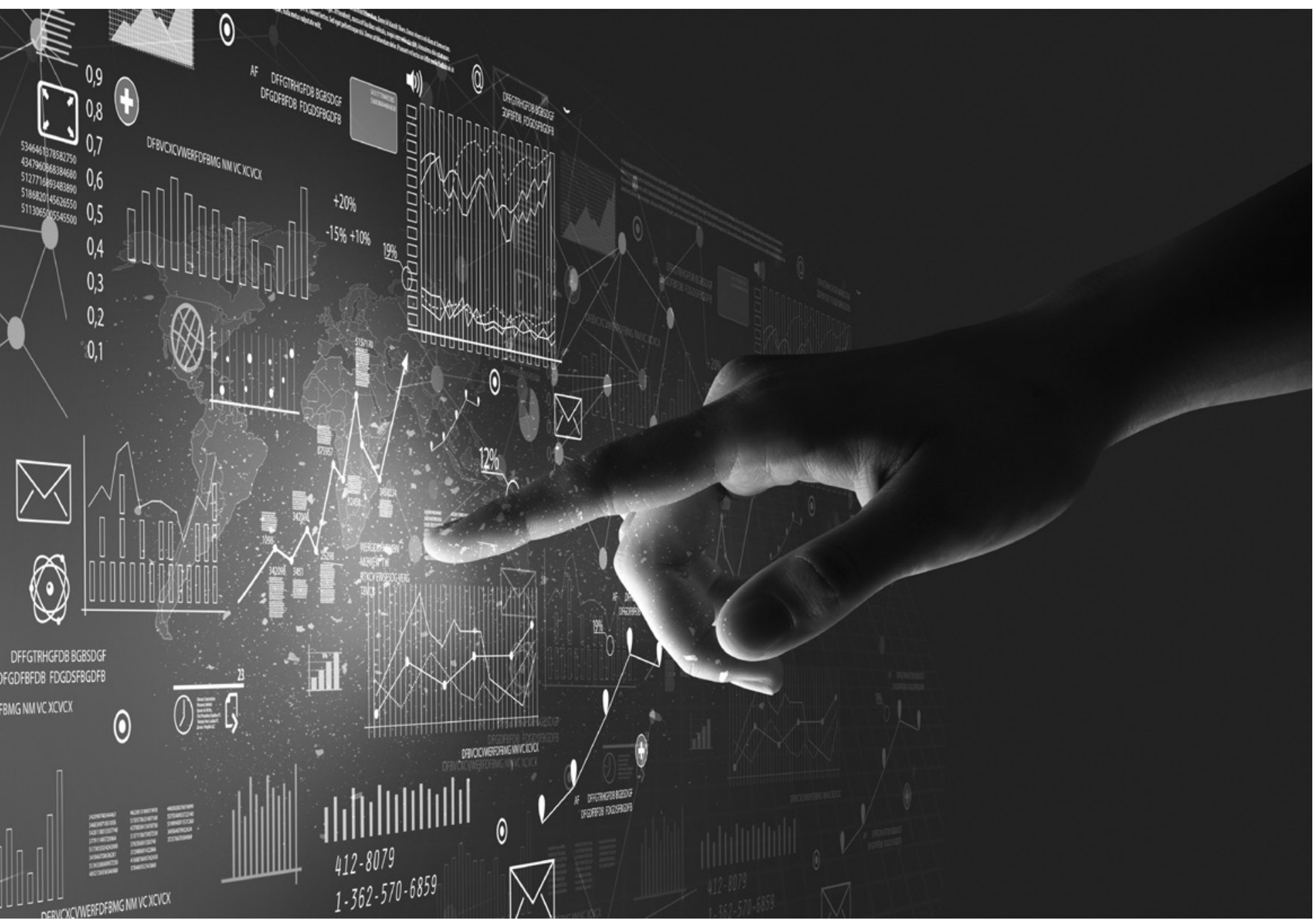


In Deutschland gibt es eine Bedingung, dass ein Therapieverfahren einer Grundorientierung zuzuordnen sei, die eine einheitliche theoretische Fundierung aufweist. Dies ist eine Grundvoraussetzung, um als eine Psychotherapierichtung überhaupt einen Wirksamkeitsnachweis erbringen zu können. In der Schweiz wurde das teils auch so gehandhabt. Das Konzept der Grundorientierung ist jedoch für Psychotherapieverfahren untauglich und überholt. Weder die Verhaltenstherapie noch die Tiefenpsychologischen Richtungen noch die Systemische Therapie und eben auch nicht die Humanistische Therapie verfügen heute über eine konsistente Grundlagentheorie, auf der sich alle ihrer Richtungen innerhalb dieser Grundorientierung beziehen. Bei der Verhaltenstherapie und der Tiefenpsychologie wie auch der Systemischen Therapie scheint das nicht zu stören, die sind anerkannt, bei der Humanistischen Therapie war dies aber ein Ablehnungsgrund. Bowe (2018) kritisiert dies scharf und zeigt auf, dass mit ungleichen Ellen gemessen wurde.

In einem Interview räumt Thomas Fydrich, Mitglied des WBP, ein, dass man künftig wohl vom Begriff der Grundorientierung bzw. dem Verfahren wegkommen müsste und Methoden auch dann anerkannt werden könnten, wenn sie empirisch genügend untersucht wurden, egal, zu welchem Verfahren sie gehörten (Fydrich et al., 2018). Kritisiert wird der WBP insbesondere deshalb, weil er sich über die letzten 20 Jahre mit dem Hinweis auf laufende Verfahren kaum mehr einem wissenschaftlichen Diskurs zu den Kriterien der Wissenschaftlichkeit gestellt hat, sondern Kraft seiner ihm übertragenen Kompetenz losgelöst vom internationalen Diskurs eigene Kriterien entwickelt hat und diese so anwendet, wie er es für gut hält. Diese Kriterien hat er laufend verengt, sodass er das an sich sinnvolle Konzept der Evidenzbasierten Medizin (evidence-based medicine; $\mathrm{EbM}$ ) soweit reduziert hat, dass er nur noch Studien im RCT-Design anerkennt und alle anderen ausschliesst. Das ist ein Missbrauch des Konzepts der EbM (Kriz, 2014; Thielen \& Kriz, 2018, S. 28), das gerade vorsieht, dass auch andersartige Studien für eine sinnvolle Evidenzbasierung zu berücksichtigen sind. Das schreibt der WBP (2010) in seinem Methodenpapier zwar auch, wendet aber seine eigenen Kriterien in den jüngsten Gutachten zur Humanistischen Psychotherapie und zur Gestalttherapie nicht an (GwG, 2018).
Das Konzept der EbM geht zurück auf eine Forschungsgruppe um David Sackett (1996). Sein Anliegen war es, in der Medizin ein Behandlungsverfahren anzuwenden, das evidenzbasiert bzw. empirisch abgesichert ist. Das Konzept der EbM sieht mindestens fünf Evidenzstufen vor, die hierarchisch gegliedert sind. Zuunterst (Stufe 5) stehen theoretische Arbeiten zum Behandlungsverfahren, ExpertInnenmeinungen und Fallserien. Auf Stufe 4 folgen Meinungen und Überzeugungen angesehener Autoritäten, auf Stufe 3 nicht experimentelle Studien wie etwa Vergleichs-, Korrelations- oder Fall-Kontrollstudien. Es soll mehr als eine vorliegen. Auf Stufe 2 gibt es zwei Möglichkeiten: Entweder soll wenigstens eine hochwertige Studie ohne Randomisierung oder wenigstens eine hochwertige Studie eines anderen Typs, eine quasi-experimentelle Studie vorhanden sein. Auf Stufe 1 rangieren die in diesem System am höchsten eingestuften Studienformen: wenigstens eine Metaanalyse auf der Basis methodisch hochwertiger, randomisierter kontrollierter Studien oder wenigstens eine ausreichend große, methodisch hochwertige RCT.

Nur in der Zusammenschau aller dieser Evidenzstufen kann von EbM gesprochen werden. Eine Reduktion auf RCTs verstösst gegen das System der EbM. Kriz (2018a, S. 262) erinnert in seiner Kritik am WBP daran, dass «bereits 1996 Sackett, Rosenberg, Gray, Hynes und Richardson sogar eindrücklich davor gewarnt» haben, «lediglich RCT-Studien irrtümlich oder missbräuchlich als EbM auszugeben». Trotzdem tut dies der WBP und solche Tendenzen gab es auch in den Schweizer ExpertInnengruppen wie auch der PsyKo. Wer Studien der unteren Evidenzstufen nicht zulässt, der verstösst gegen die Prinzipien der EbM. Ausserdem zeigt sich, wie der deutsche Standard von mindestens drei Level-1-Studien deutlich über dem liegt, was EbM fordert.

Die Evidenzstufe 1 gilt als sogenannter Goldstandard. Dieser Begriff hat zwei Quellen: Er bezieht sich einerseits auf einen Pharmakologen, Harry Gold, der in der Entwicklung des randomisierten, doppelblinden Placebo-Kontrollgruppendesigns, das als Goldstandard für die Wirksamkeit von Psychotherapie und Medikamenten geworden ist, sehr engagiert war. Andererseits erinnert der Begriff an Gold als wertvolles Metall (Wampold et al., 2018).

Hart kritisiert wird dieser Goldstandard für die Psychotherapie auch deshalb, weil dieses Design 
aus der experimentellen Psychologie stammt, in einem Verständnis, dass die Person des Therapeuten oder der Therapeutin austauschbar sein sollte, also höchstens als Störvariable zu kontrollieren ist. Das mag, wie Kriz (2014) zeigt, für den Ansatz der frühen Verhaltenstherapie passen, nicht aber für psychodynamische und humanistische Verfahren. Er sieht den Wert der EbM in der Prüfung von ganzen Therapieprogrammen, nicht aber im Prüfen von Therapieverläufen, wo es andere Designs braucht, um Prozesse der Veränderung zu erforschen. Er fordert für die Zukunft, dass die Forschungsmethoden den verschiedenen Verfahrens-Ansätzen zu entsprechen hätten, statt dass diese sich, um den Kriterien des WBP zu entsprechen, ins Prokrustesbett des RCT-Designs zu legen hätten und dabei ihr Forschungsinteresse so verfälschen müssten, dass Artefakte rauskämen, nicht aber das, was wesentlich wäre.

Die ForscherInnengruppe der Praxisstudie ambulante Psychotherapie Schweiz (PAP-S) hat das RCT-Design als Goldstandard der Psychotherapieforschung ebenfalls stark kritisiert und begründet, weshalb ein naturalistisches Studiendesign vorzuziehen sei (Tschuschke et al., 2009). Das bereits erwähnte Gespräch mit Fydrich (Fydrich et al., 2018) begrüsst Kriz (2018b, S. 5) als Zeichen der Rückkehr zu einem wissenschaftlichen Dialog und hofft auf eine Lernbereitschaft des WBP, denn «längst nicht alle Fragen lassen sich mit dem experimentellen Ansatz, der der RCT-Logik zugrunde liegt, angehen».

Er fordert, dass der WBP möglichst bald neu zu besetzen sei, auch hinsichtlich des neuen Gesetzes in Deutschland: Er sei einseitig von VerhaltenstherapeutInnen besetzt. Es seien Vertreter aller vier Grundrichtungen einzubeziehen. Der neue WBP solle eine Revision des Methodenpapiers an die Hand nehmen mit dem Ziel, die Pluralität von Forschungsdesigns in der Scientific Community zu berücksichtigen und sich zumindest an allen Kriterien der EbM zu orientieren, er solle längerfristig aber auch neuere Forschungsansätze in der Einzelfallforschung etc. berücksichtigen - vor allem wenn Studien mit entsprechenden Designs zu bewerten sind. Und es sei zu überlegen, ob nicht zumindest ein oder eine MethodikerIn und WissenschaftstheoretikerIn im WBP Platz haben sollte.

In der Schweiz werden in den nächsten Monaten die Erfahrungen der Akkreditierungen aus- gewertet und danach wohl manche Standards zur Akkreditierung neu oder präziser gefasst werden. Die ASP, die übrigen Verbände und die akkreditierten Institute tun gut daran, diese Entwicklung im Auge zu behalten und mitzuwirken. Auch hierzulande gilt es dafür einzutreten, dass eine Pluralität der Forschungsdesigns berücksichtigt sowie die EbM nicht ad absurdum geführt wird, und es gilt darauf zu achten, dass die PsyKo nicht einseitig von Verhaltenstherapie-ProfessorInnen zusammengesetzt bleibt, sondern VertreterInnen aus allen vier Grundorientierungen und der Körperpsychotherapie Einsitz erhalten.

\section{Literatur}

AkkredV-PsyG (2013). Verordnung des EDI über Umfang und Akkreditierung der Weiterbildungsgänge der Psychologieberufe vom 25.11.2013. Stand: 01.01.2016 (935.811.1).

Bowe, N. (2018). Wie wissenschaftlich entscheidet der Wissenschaftliche Beirat Psychotherapie? Projekt Psychotherapie, (3), 19-21.

Fydrich, T., Sartorius, A. \& Schweizer-Köhn, E. (2018). «Ich bin davon überzeugt, dass Gesprächspsychotherapie allein nicht hinreichend wirksam ist». Gespräch. Projekt Psychotherapie, (3), 30-32.

GwG (2018). Gutachten des Wissenschaftlichen Beirats Psychotherapie auch formal fehlerhaft. https://www. gwg-ev.org/presse/gutachten-des-wissenschaftlichenbeirats-psychotherapie-auch-formal-fehlerhaft (08.04.2019).

Kriz, J. (2014). Wie evident ist Evidenzbasierung? Über ein gutes Konzept - und seine missbräuchliche Verwendung. In S. Sulz (Hrsg.), Psychotherapie ist mehr als eine Wissenschaft. Ist hervorragendes Expertentum durch die Reform gefährdet? (S. 154185). München: CIP.

Kriz, J. (2015). Psychotherapieforschung - und ihre Beschränkung durch einen schulenspezifischen Bias. Resonanzen, 3(2), 101-113.

Kriz, J. (2018a). Gutachten des Wissenschaftlichen Beirats Psychotherapie zu Humanistischen Psychotherapie - Und wie geht es nun weiter? Psychotherapeutenjournal, (3), 257-264.

Kriz, J. (2018b). Rückkehr zum wissenschaftlichen Diskurs? Familiendynamik, 43(4), 2-5. https://doi. org/10.21706/fd-43-4-0000

PsyG (2011). Psychologieberufegesetz vom 18.03.2011. Stand: 01.01.2018 (SR 935.81). 
Schulthess, P. (2018a). Gutachten des deutschen Wissenschaftlichen Beirates zur Humanistischen Psychotherapie. à jour! Psychotherapie-Berufsentwicklung, 4(1), 16-18.

Schulthess, P. (2018b). Deutschland: Gutachten des WBP zur Gestalttherapie. à jour! PsychotherapieBerufsentwicklung, 4(2), 15.

Sackett, D.L. et al (1996). Evidence based Medicine: What it is and what it isn't. British Medical Journal, 312, 71f. https://doi.org/10.1136/bmj.312.7023.71

Thielen, M. \& Kriz, J. (2018). Willkürlich und intransparent. Zur Ablehnung der humanistischen Psychotherapie durch den Wissenschaftlichen Beirat aus Sicht der AGHPT. Projekt Psychotherapie, (3), 27-29.

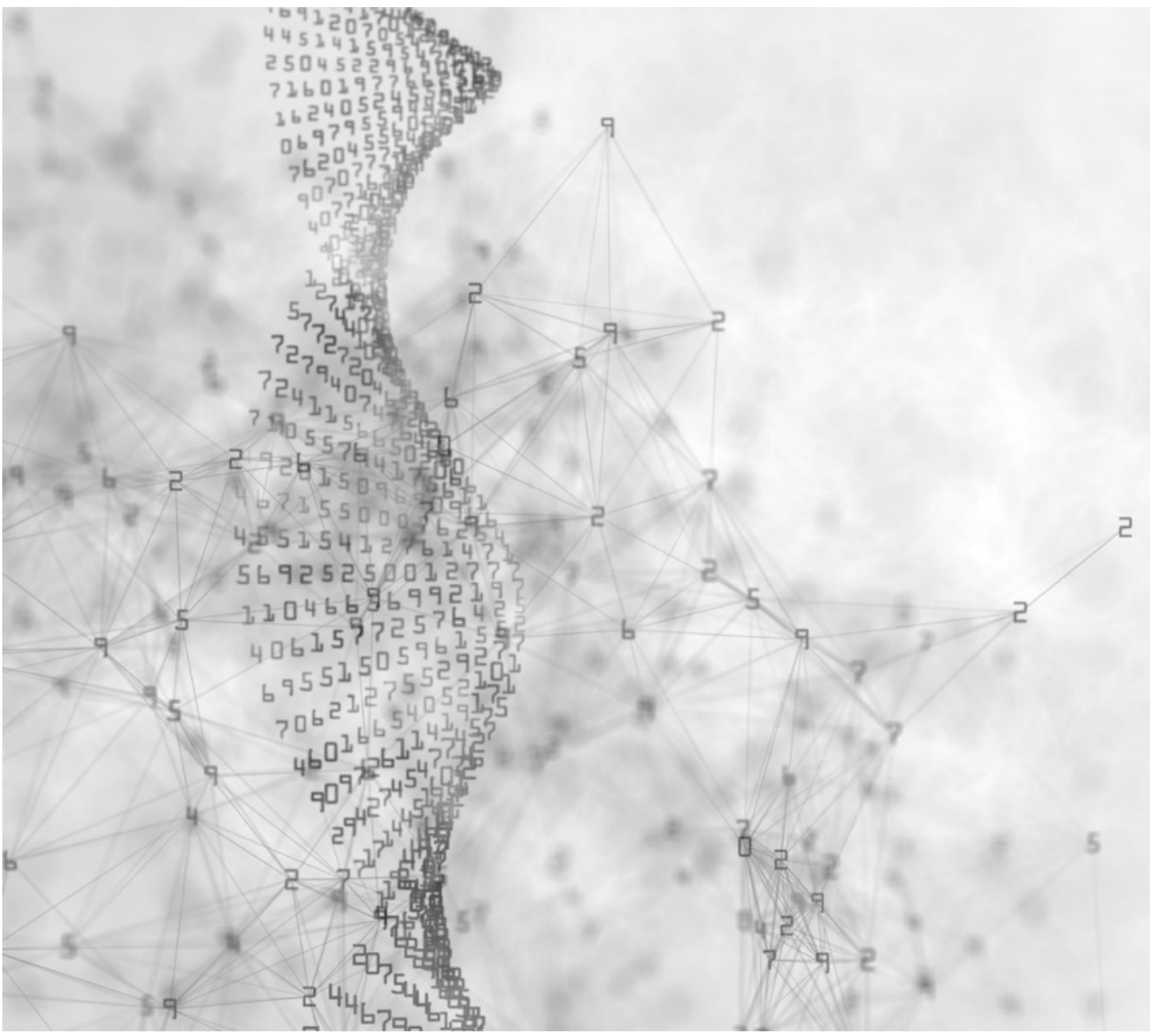

Tschuschke, V., Crameri, A., Koemeda, M., Schulthess, P., von Wyl, A. \& Weber, R. (2009). Psychotherapieforschung - Grundlegende Überlegungen und erste Ergebnisse der naturalistischen Psychotherapie-Studie ambulanter Behandlungen in der Schweiz (PAP-S). Psychotherapie Forum, 17(4), 160-176.

Wampold, B.E., Imel, Z.E. \& Flückiger, C. (2018). Die Psychotherapie-Debatte. Was Psychotherapie wirksam macht. Bern: Hogrefe.

WBP (2010). Methodenpapier Version 2.8. vom 20.09.2010. https://www.wbpsychotherapie.de/ methodenpapier (08.04.2019).

Peter Schulthess ist Vorstandsmitglied der ASP und Vorsitzender des Wissenschaft- und Forschungs-Komitees der EAP. 